OPEN ACCESS

Edited by: Hassane Zouhal,

University of Rennes 2 - Upper Brittany, France

Reviewed by:

Gaetano Raiola,

Università degli Studi di Salerno, Italy

Giovanni Messina

University of Foggia, Italy

*Correspondence:

Luca P. Ardigò

luca.ardigo@univr.it

Specialty section: This article was submitted to

Exercise Physiology,

a section of the journal

Frontiers in Physiology

Received: 27 April 2018

Accepted: 17 July 2018

Published: 03 August 2018

Citation:

Padulo J, Nikolaidis PT, Cular D, Dello lacono A, Vando $S$, Galasso $M$,

Lo Storto D and Ardigò LP (2018) The Effect of Heart Rate on Jump-Shot

Accuracy of Adolescent Basketball

Players. Front. Physiol. 9:1065.

doi: 10.3389/fphys.2018.01065

\section{The Effect of Heart Rate on Jump-Shot Accuracy of Adolescent Basketball Players}

\author{
Johnny Padulo 1,2, Pantelis T. Nikolaidis ${ }^{3}$, Drazen Cular', Antonio Dello lacono4, \\ Stefano Vando', Maria Galasso', Dario Lo Storto' ${ }^{1}$ and Luca P. Ardigò ${ }^{4,5 *}$ \\ ${ }^{1}$ Faculty of Psychology, eCampus University, Novedrate, Italy, ${ }^{2}$ Faculty of Kinesiology, University of Split, Split, Croatia, \\ ${ }^{3}$ Department of Physical and Cultural Education, Hellenic Army Academy, Athens, Greece, ${ }^{4}$ Zinman College of Physical \\ Education and Sport Sciences, Wingate Institute, Netanya, Israel, ${ }^{5}$ Department of Neurosciences, Biomedicine \\ and Movement Sciences, School of Exercise and Sport Science, University of Verona, Verona, Italy
}

Basketball is a team sport, where fundamental skills - fundamentals - are key determinants for success. Jump-shot (JS) is a basketball fundamental used frequently during game. It is interesting to spread light on the relationship between effort intensity and JS ability. Study aim was to investigate different heart rates (HRs) effect on JS accuracy (JS\%) in 22 male youth (15.7 \pm 0.9 years) players. Experimental sessions consisted of $10 \mathrm{JSs}$ from five spots $5 \mathrm{~m}$ from basket at three different HRs: rest (OHR) and after warm-up (50\% [50HR] and $80 \%$ maximal HR [80HR]). Analysis of variance showed differences in JS\% over sessions (42.27 $\pm 14.78 \%$ at OHR, $38.18 \pm 10.53 \%$ at $50 \mathrm{HR}$, and $30.00 \pm 16.62 \%$ at $80 \mathrm{HR} ; P=0.018)$. Least significant difference test did not show any significant difference between 50HR and OHR JS\% $(P=0.343)$, while $80 \mathrm{HR}$ elicited significantly lower values with respect to both $\mathrm{OHR}(P=0.006)$ and $50 \mathrm{HR}$ $(P=0.049)$. Study provided practical indications on maintaining high $\mathrm{JS} \%$ : preliminary warm-up (even if injury-protecting) does not improve $\mathrm{JS} \%$, because between $50 \mathrm{HR}$ and OHR difference was not significant; and $80 \mathrm{HR}$ significantly decreases JS\%. Therefore, to maximize JS scoring players have to rest as much as possible during game-play pauses, and coaches should manage timeouts and substitutions accordingly, especially during final minutes of close games.

Keywords: basketball, bpm, fatigue, motor control, task performance and analysis, team sports

\section{INTRODUCTION}

Basketball is a team sport, where performance depends on physical, physiological, psychological, technical and tactical characteristics. Among these characteristics, an increased scientific interest has been observed in the profiling of physical and physiological characteristics with regards to age and playing position (Nikolaidis et al., 2014, 2015). Accordingly, several studies have been conducted recently to develop sport-specific exercise tests (e.g., including uni- or multi-directional repeated sprints) in order to match the metabolic demands and activity patterns of basketball (Attene et al., 2015b, 2016; Padulo et al., 2015b, 2016; Zagatto et al., 2017), whereas other studies have examined the effectiveness of sport-specific training interventions (Attene et al., 2014, 2015a). Although the physical, physiological and psychological characteristics, tactical and nutritional aspectis, commonly considered as key components for successful basketball performance, have 
already been investigated, less information is available on technical skills, such as the shooting task (Padulo et al., 2015a; Ardigò et al., 2018). It has been supported (Okazaki et al., 2015) that the ability to shoot a jump-shot (JS) is a major component of sport performance. Moreover, it has been shown that players perform shooting more often under conditions of low-pressure and fast movements (Csapo et al., 2015). Professionals can shoot from longer distances than their amateur counterparts, and perform more team tactics in order to be in position where, likely, a relatively lower defensive pressure exists (Ibáñez et al., 2009). With regards to playing position, point guards and power forward perform shooting more frequently and successfully in free-throw (FT) and two-point shots, and point guards shoot more frequently successfully in three-point shots (Ortega et al., 2006).

The variables that affect ball trajectory have been well studied (Okazaki et al., 2015). When deciding to shoot, players consider four factors: physical defensive pressure, rebounding issues, defensive balance, and shooting distance (Llorca-Miralles et al., 2013). Time remaining before the shot clock expires might be another factor (Skinner, 2012). Shooting accuracy can be influenced by defensive intensity, and to a lesser degree by how many defenders are guarding the shooter (Csapo and Raab, 2014).

The abovementioned studies on factors influencing shooting performance have improved the scientific understanding about the key requisites targeted as fundamental for shooting performance accuracy. However, less data exist about the role of fatigue, induced by different exercise intensities, on JS\%. Just as a reference, in the 2015-16 season (regular season and playoffs) the NBA champions Cleveland Cavaliers shot - contested by players from the opposing team - JSs from a distance of between 16 and 17 feet (between 4.88 and $5.18 \mathrm{~m}$ ) in the first and last $2 \mathrm{~min}$ of the regular quarters with JS percentages (JS\%) of 44.3 and $28.3 \%$, respectively ${ }^{1}$. Clearly, directly from the above reference, it is not possible to clear which is the cause of the JS\% decrease, be it fatigue, game intensity, time pressure... However, the JS is surely a popular basic shot that might be used in various conditions during a match (e.g., after continuous match-play or after a time-out). Information concerning the role of fatigue on JS\% might be of practical interest for a large number of strength and conditioning coaches. As there have been no previous studies on the effects of exercise intensity on JS\%, researchers might employ such data as reference for future research on this team sport. Moreover, strength and conditioning coaches could apply this information to prescribe proper training regimes to optimize shooting performance.

Basketball became one of the most popular team sports worldwide, but relatively few papers have been published so far about the determinants of shooting accuracy despite its great relevance for successful performance. Present study wished to provide field information on the relationship between fatigue and shooting ability and practical knowledge for players' skills development trainers. Consequently, the objective of this study

${ }^{1}$ BASKETBALL REFERENCE, http://www.basketball-reference.com/play-index/ plus/shot_finder.cgi was to investigate the role of different heart rate (HR) conditions on JS\%. We adopted the research hypothesis that an increased HR would result in decreased JS\%.

\section{MATERIALS AND METHODS}

\section{Participants}

Twenty-two adolescent male basketball players (age $15.7 \pm 0.9$ years; height $179.0 \pm 6.9 \mathrm{~cm}$; mass: $66.0 \pm 9.6 \mathrm{~kg}$; BMI $20.6 \pm 2.0 \mathrm{~kg} / \mathrm{m}^{-2}$; training experience $8.4 \pm 3.0$ years), players of 7 Laghi Gazzada team, volunteered for the present study. All participants were training regularly during weekdays and were playing official match every weekend. To be eligible for this study, a participant should have attended a minimum of $85 \%$ of all team's exercise programs, had a valid sport medical certification and did not report any illness or injury. This study was performed considering the recommendations of the Code of Ethics of the World Medical Association. The protocol was approved by the University of Split Ethics Committee. All participants' parents/guardians provided written informed consent in accordance with the Declaration of Helsinki.

\section{Protocol}

Participants were instructed to abstain from drinking alcohol or beverages containing caffeine for $24 \mathrm{~h}$, and did not eat for $3 \mathrm{~h}$, prior to testing, in order to decrease their possible effect on the testing outcome. Each participant performed all trials in the same time period and under the same climatic conditions of the testing days $\left(15: 00-17: 00,22.5 \pm 0.6^{\circ} \mathrm{C}\right.$ temperature, and $59.2 \pm 2.4 \%$ relative humidity), to minimize any effect of circadian variation. All tests were carried out on an indoor basketball court.

This experimental study was approached through a crossover observational design (i.e., all players shot their JSs over different sessions in different days). We used $\mathrm{HR}$ as the independent variable and JS\% as the dependent variable. In the first session, the participants carried out a Yo-Yo Intermittent Recovery test level 1 (Yo-Yo IR1; Castagna et al., 2008) to measure maximal heart rate $\left(\mathrm{HR}_{\mathrm{MAX}}\right)$. One week later, the participants executed three randomized shooting testing sessions with a 1 -h rest between two consecutive sessions. All sessions included 10 consecutive JSs (regular ball - Molten gf7, 600 gr.) from five different commonly chosen field spots, at a distance of $5 \mathrm{~m}$ from the (regular) basket (Figure 1, two counter-clockwise laps), at self-selected gameplay pace, at different HRs: at rest without warm-up (0HR), immediately after warm-up with $\mathrm{HR}$ at $50 \% \mathrm{HR}_{\mathrm{MAX}}(50 \mathrm{HR}$; i.e., a reasonable post-warm-up HR value; Garrett and Kirkendall, $2000)$, and $80 \% \mathrm{HR}_{\mathrm{MAX}}(80 \mathrm{HR}$; i.e., a reasonable actual play HR value; McInnes et al., 1995). Two balls were used, and two rebounders ensured an accurate ball pass to the shooter at each different spot. The shooting distance was kept constant in order to eliminate its specific effect on shooting kinematics (Miller and Bartlett, 1996). The same procedure was repeated 1 week later to evaluate the measures' reliability. Each HR (continuously monitored with Cardio-SuuntoTM) was achieved by increasing the intensity of the warm-up shuttle running $(15+15 \mathrm{~m}$ with $180^{\circ}$ changes of direction) chosen as an ecological way to 


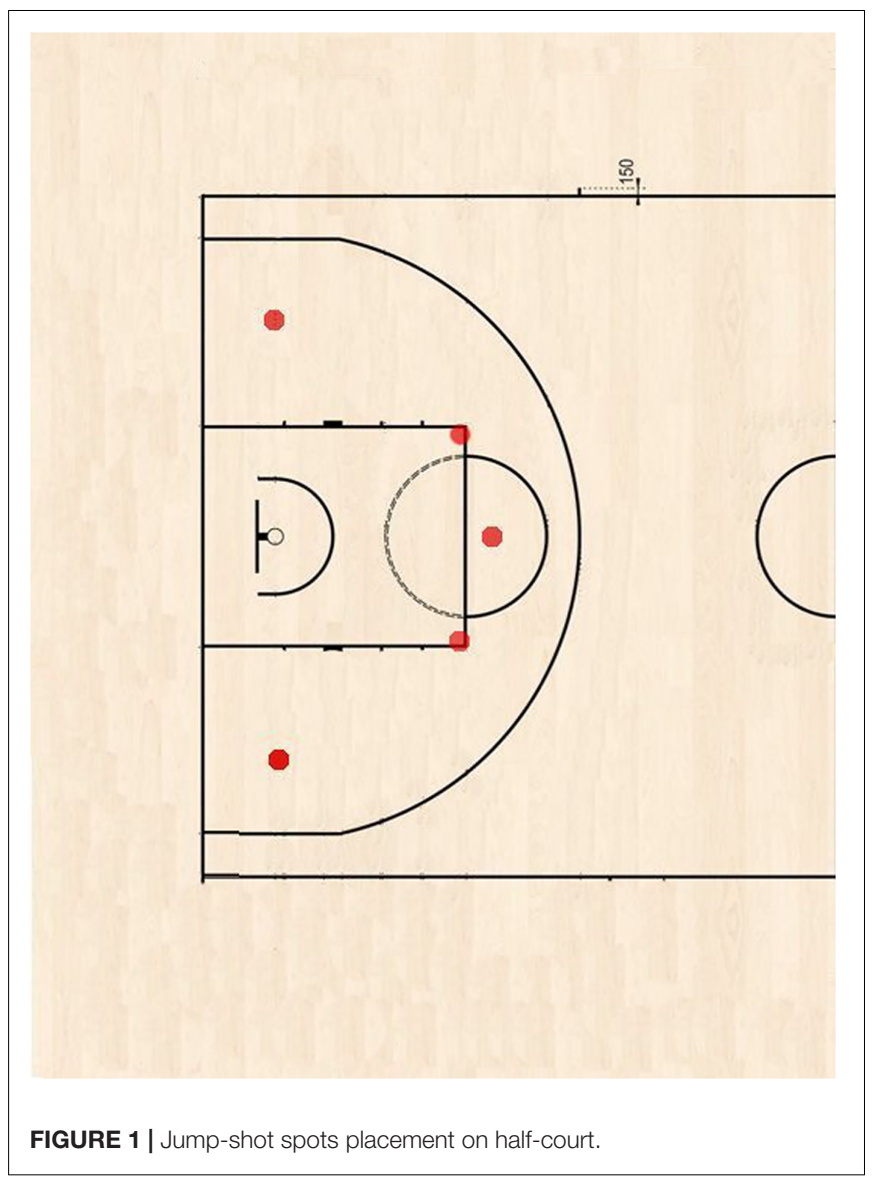

simulate fatigue provided by game-play. Briefly, the participants needed to run on average 540 and $1620 \mathrm{~m}$ to achieve 50 and $80 \% \mathrm{HR}_{\mathrm{MAX}}$, respectively.

\section{Statistical Analysis}

Descriptive statistics (mean \pm SD and SE) were calculated for the Yo-Yo IR1, and the $0 \mathrm{HR}, 50 \mathrm{HR}$, and $80 \mathrm{HR}$ (effective $\% \mathrm{HR}_{\mathrm{MAX}}$ and measured JS\%, i.e., successful JSs/total JSs\%) test results, to present the data. Statistical analyses were carried out using SPSS 17.0 (SPSS Inc., Chicago, IL, United States). Normality of the data was tested with the Shapiro-Wilk test and homogeneity of variances was examined using the Bartlett's test. Intra-class Correlations Coefficient (ICC) estimated the reliability of the $0 \mathrm{HR}, 50 \mathrm{HR}$, and $80 \mathrm{HR}$ tests. ICC evaluation criterium is: $<0.4$ poor, 0.4-0.75 fair to good, and $>0.75$ excellent (Fleiss, 1991). Furthermore, a one-way within-participants analysis of variance (ANOVA) examined differences among the three test levels (0HR, 50HR, and 80HR) with the post hoc Least Significant Difference test (LSD). Cohen's $d$ effect sizes (ESs) obtained in each statistical analysis were shown and interpreted as proposed by Hopkins ${ }^{2}$, with ES $<0.2$ considered as trivial, $0.2-0.5$ small, 0.6-1.1 moderate, 1.2-1.9 large, and $>2$ very large. Statistical significance was set at a $P \leq 0.05$.

\footnotetext{
${ }^{2}$ A New View of Statistics, http://www.sportsci.org/resource/stats/
}

\section{RESULTS}

The ICC demonstrated a good reliability at $0 \mathrm{HR}$ (0.89), $50 \mathrm{HR}$ (0.92), and 80HR (0.95). Yo-Yo IR1-induced HR MAX was $196.8 \pm 6.6 \mathrm{bpm}$, corresponded to a total distance of $1724 \pm 671 \mathrm{~m}$, and an end running speed of $18.3 \pm 1.9 \mathrm{~km} / \mathrm{h}$. ANOVA showed differences of HR among the three applied exercise conditions (Figure $2 ; F_{(1,20)}=4775.001$ with $P=0.000$ ): $0 \mathrm{HR}(58.3 \pm 1.9 \mathrm{bpm}), 50 \mathrm{HR}(97.8 \pm 3.1 \mathrm{bpm})$, and $80 \mathrm{HR}$ $(156.0 \pm 4.9 \mathrm{bpm})$. Each shooting session lasted less than $1 \mathrm{~min}$ and did not contribute to increase starting HR more than 5\%. Similarly, ANOVA showed differences of JS\% over the three exercise conditions (Figure 2; $F_{(1,20)}=4.257$ and $P=0.018$ ), with a total of 660 JSs shot. JS\% in the three exercise conditions was $42.27 \pm 14.78 \%$ at $0 \mathrm{HR}, 38.18 \pm 10.53 \%$ at $50 \mathrm{HR}$, and $30.00 \pm 16.62 \%$ at $80 \mathrm{HR}$. The LSD test did not show any significant difference between 50HR and 0HR JS\% $(-10 \%$, ES $=-0.28$, small, and $P=0.343)$, whereas $80 \mathrm{HR}$ elicited significantly lower values of JS\% compared to $0 \mathrm{HR}$ and $50 \mathrm{HR}$ $(-29$ and $-21 \%$ with $\mathrm{ES}=-0.83$ and -0.78 (moderate), with $P=0.006$ and $P=0.049$, respectively).

\section{DISCUSSION}

In the present study the effect of exercise intensity on JS\% was examined. Three conditions of exercise intensity were considered: rest (0HR), warm-up (50HR), and exercise simulating the average intensity of a basketball game (80HR). The main findings were that (a) JS\% was lower in the 80HR than in the 0HR $(-29 \%)$ and 50HR $(-21 \%)$ conditions, and (b) no difference between 50HR and 0HR was observed.

To the best of our knowledge, the present study is the first to specifically examine the variation in JS\% induced by exercise intensity. These results are in line with those of previous research on the effect of exercise intensity on FT (Padulo et al., 2015a; Mokou et al., 2016) and three-point shot (3S) accuracy (Ardigò et al., 2018), which showed a significant reduction in shot accuracy at $80 \mathrm{HR}$ compared to $0 \mathrm{HR}$ and $50 \mathrm{HR}$. Thus, exercise intensity exerted a similar effect on the $3 \mathrm{~S}$, JS, and FT, yet, a difference can be identified between the $3 \mathrm{~S}$ and JS compared with the FT. Namely, FT is not a game-play flow-driven basketball fundamental, given that it is basically shot without any defence pressure and during a game-play pause. In contrast, the $3 \mathrm{~S}$ and JS are fundamentals performed throughout a much longer and complex game-play action, potentially involving the contribution of the entire playing team (e.g., by means of a plan), and with the opposition of one or more defenders. Therefore, game-play pace surely has some effect on $3 \mathrm{~S}$ and JS performance, and real gameplay study simulation may not be as effective here as in the FT simulation.

However, our outcomes - simply in terms of exercise intensity effect on JS\% - are similar to those shown by previous studies on the effect of fatigue on passing accuracy (Lyons et al., 2006; Ahmed, 2013). Although the designed fatiguing protocols in these studies were different from the current study's protocol 


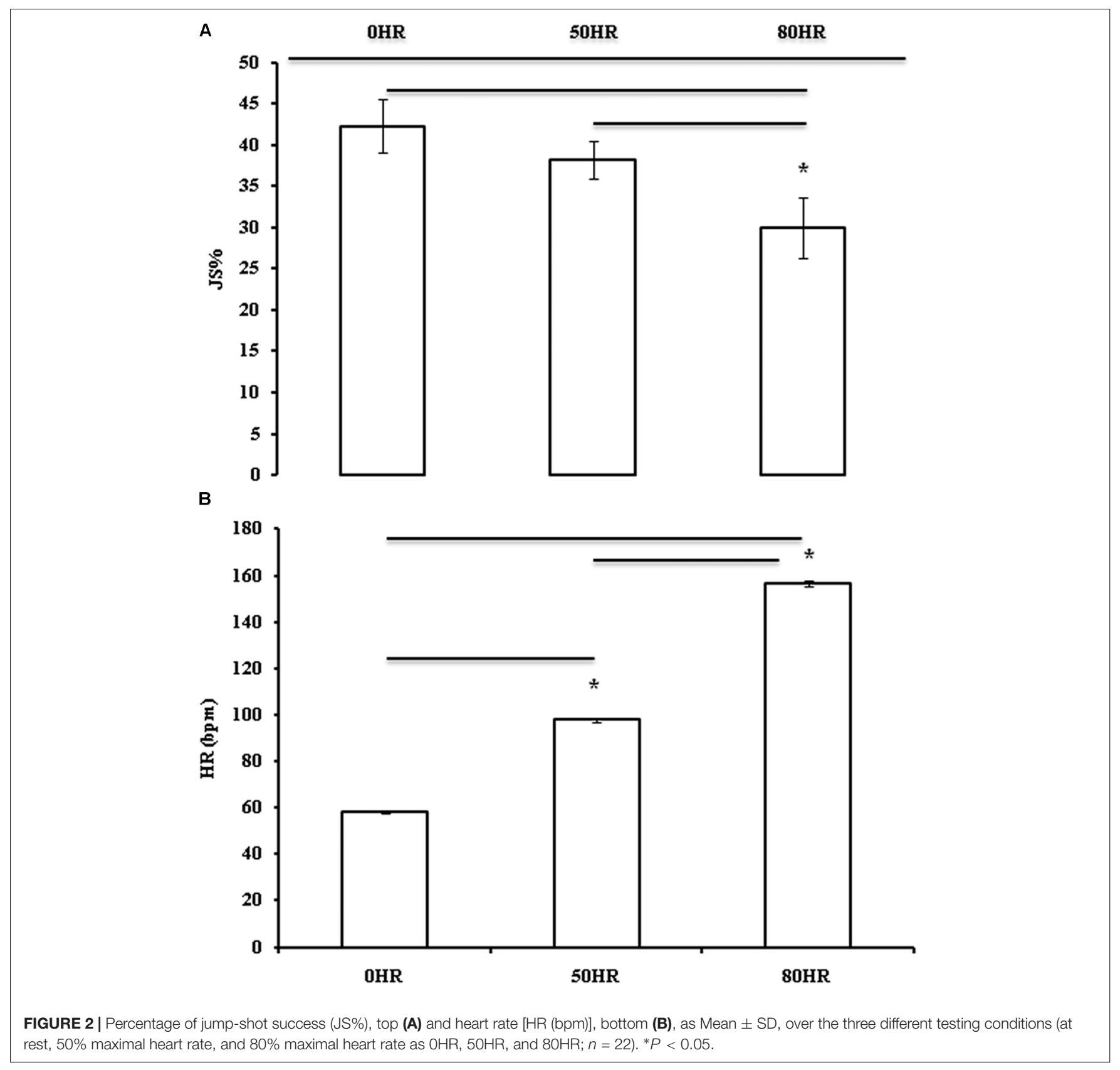

(i.e., gym machines-based vs. intermittent shuffle running), the decrease in technical skill accuracy ranged from 15 to $30 \%$, thus similar to that observed about JS\%. To date, the effect that fatigue has on the performance of the basketball JS is not clear. From a biomechanical viewpoint, an appropriate whole body movement organization - from the lower body segments, through the trunk, and up to the ball-releasing segment of the hand-fingers complex, is required for the development of a shooting movement pattern that results in a successful shot. The JS represents a complex sequence of proximal to distal force and moment interrelations, with the goal of transferring energy from the lower extremity and trunk up and through the upper extremity kinetic chains. Finally, the upper extremity must effectively control and transfer these forces in order to sustain an optimal and accurate action. Therefore, a possible explanation of the deteriorating effect of fatigue on shooting accuracy might be the alterations of some kinematic characteristics occurring in the involved movements (whose specific description goes beyond the aim of this study). The sensorimotor system is responsible for providing the awareness, coordination, and feedback to maintain intra-joint stability and inter-joint coordination during complex movements (Lephart et al., 2000; Gheller et al., 2015). Evidence indicates that the sensorimotor system may be compromised by fatigue. Fatigue seems to hamper the sensorimotor system function in a way similar to joint injury. As a consequence, sensory motor deficits may result in the inability to appreciate 
and maintain the ideal mechanics that should accompany a prolonged shooting task (Forestier and Nougier, 1998). It has been shown that optimal timing, sequence, and amplitude of the muscle activation and joint movements are required for JS-like shot performance, even when the task is performed by different subjects (Forestier and Nougier, 1998). Moreover, some movement constraints (e.g., constraining the trunk segment) only slightly disrupt the pattern of variables used to describe coordination (e.g., muscle activation; Eloranta, 1996). Indeed, breakdowns in shooting mechanics may represent reasonable limiting factors for successful performance (Okazaki et al., 2015). Accordingly, studies that analysed the effect of fatigue on motor skills such as the vertical jump (Rodacki et al., 2002) revealed that when the primary muscles responsible for the jumping movement were fatigued, the movement resulted in greater involvement of the synergistic muscles. Thus, these results suggest that fatiguing the muscles involved in basketball shooting (e.g., fatiguing the lower limbs that perform the jump or the upper limbs that perform the ball release) may result in a decline in the player's level of performance. For instance, it has been shown that fatigue induces a decrease in the height of the shoulder axis and of the wrist (Erculj and Supej, 2009). The underlying physiological mechanisms leading to the decrease in JS\% at $80 \mathrm{HR}$ due to the fatiguing protocol may be attributed to various factors related to the muscle and blood metabolites. From a methodological perspective, the fatiguing protocol designed in our study to induce the targeted physiological responses (e.g., 80HR) implied a setup including intermittent low/high-intensity shuffle running bouts similar to those included in the Yo-Yo test (Castagna et al., 2008). Therefore, performing shuffle running repeatedly, with the inclusion of acceleration, deceleration, and change of direction actions, may have increased the chances for the players to incur a short-term fatigue-related adaptation that, in turn, may have altered the mechanics of the JS performance and hence the scoring accuracy.

A determining factor worth considering for the interpretation of the results of our study is the experience level of the involved population. Previous studies comparing the movement patterns of the FT and JS across players of different levels have identified an inter-individual variability, with technical performances being more variable in beginner players and less evident in experienced ones (Button et al., 2003; Okazaki et al., 2007). Specifically, novice players seem to constrain the degrees of freedom of their joint movements during the release phase of JS (Temprado et al., 1997), in order to make straightforward the controlling tasks imposed on the central nervous system during this phase (Newell and Vaillancourt, 2001; Okazaki et al., 2007). In turn, this constraining organizational pattern of motion prevents the beginner player from using a wider range of choices that have been shown in the game-play of experienced players. Consequently, sport experience is an aspect that might influence inter-individual differences when performing a JS under fatigued conditions. Hence, the evident greater variability observed among novice athletes in performing technical skills associated with fatigueinduced impairments on shooting accuracy may have produced a cumulative negative effect on the JS performance, as reported in our data in the $80 \mathrm{HR}$ condition. On the other hand, the age and sport experience $(\sim 16$ and $\sim 8$ years, respectively) of the basketball players who represent the sample of this study may also constitute a specific limitation of the findings, given that the conclusions should be translated with caution to other age and performance groups. A previous investigation on the effect of exercise intensity on passing accuracy in basketball reported that passing performance decreased with increasing fatigue, in both novice and expert basketball players. However, the expert players showed less deterioration of performance (Lyons et al., 2006). Players' young age and sport experience might have impacted study results in terms of specific age and training dependent heart responses (Francavilla et al., 2018). Another potentially confounding factor we neglected was players' specific psychological genes-driven answer to study protocol (Sessa et al., 2011; Petito et al., 2016).

Further study limitations are the following ones. We focused as metabolic power proxy only on pre-set HR values and not on Karvonen's HR reserve ones (Karvonen et al., 1957) and/or rates of perceived exertion (Borg, 1982). Our testing conditions were very controlled, whereas real game-play might be by its nature chaotic with different level opponents, many lead changes, opposing fans' behaviors, etc. Final limitation was that we neglected playing position differences.

Increased metabolic conditions, as witnessed by increasing HRs, decrease JS\%. Therefore, players should be encouraged to rest as much as possible during game-play pauses, and coaches should support this resting strategy by calling timeouts and managing substitutions wisely, especially during the final minutes of close games. Players should perform tailored physical training to condition their physical capacity and technical skills simultaneously. Our findings, obtained by a means of a nongame-play protocol, should be taken into account with caution, even if they provide indications similar to those provided by similar studies on other basketball fundamentals. Future research could investigate the JS in greater depth in other age and sport experience groups, and could repeat our approach in investigating other team sports fundamentals (e.g., volleyball blocking).

\section{CONCLUSION}

The findings of the present study have implications for professionals working with basketball players. Based on these results, it is recommended that coaches focus on exercises using the JS under metabolic conditions simulating a basketball game. Our results suggest two practical applications to maintain high JS\%: (1) preliminary warm-up (even if needed to protect the body against injuries) does not improve JS accuracy, since the between $50 \mathrm{HR}$ and $0 \mathrm{HR}$ difference was not significant; and (2) $80 \mathrm{HR}$ decreases JS accuracy significantly, therefore providing scientific support for the usual behavior of players who always aim to rest as much as possible during game-play pauses, and for coaches, who support players' behavior by calling timeouts and managing substitutions accordingly, especially during the final minutes of close games. 


\section{AUTHOR CONTRIBUTIONS}

All authors listed have made a substantial, direct and intellectual contribution to the work, and approved it for publication.

\section{REFERENCES}

Ahmed, T. (2013). The effect of upper extremity fatigue on grip strength and passing accuracy in junior basketball players. J. Hum. Kinet. 37, 71-79. doi: 10.2478/hukin-2013-0027

Ardigò, L. P., Kuvacic, G., Dello Iacono, A., Dascanio, G., and Padulo, J. (2018). Effect of heart rate on basketball three-point shot accuracy. Front. Physiol. 9:75. doi: 10.3389/fphys.2018.00075

Attene, G., Iuliano, E., Di Cagno, A., Calcagno, G., Moalla, W., Aquino, G., et al. (2015a). Improving neuromuscular performance in young basketball players: plyometric vs. technique training. J. Sports Med. Phys. Fitness 55, 1-8.

Attene, G., Laffaye, G., Chaouachi, A., Pizzolato, F., Migliaccio, G. M., and Padulo, J. (2015b). Repeated sprint ability in young basketball players: one vs. two changes of direction (Part 2). J. Sports Sci. 33, 1553-1563. doi: 10.1080/ 02640414.2014 .996182

Attene, G., Nikolaidis, P. T., Bragazzi, N. L., Dello Iacono, A., Pizzolato, F., Zagatto, A. M., et al. (2016). Repeated sprint ability in young basketball players (part 2): the chronic effects of multidirection and of one change of direction are comparable in terms of physiological and performance responses. Front. Physiol. 7:262. doi: 10.3389/fphys.2016.00262

Attene, G., Pizzolato, F., Calcagno, G., Ibba, G., Pinna, M., Salernitano, G., et al. (2014). Sprint vs. intermittent training in young female basketball players. J. Sports Med. Phys. Fitness 54, 154-161.

Borg, G. A. (1982). Psychophysical bases of perceived exertion. Med. Sci. Sports Exerc. 14, 377-381. doi: 10.1249/00005768-198205000-0 0012

Button, C., MacLeod, M., Sanders, R., and Coleman, S. (2003). Examining movement variability in the basketball free-throw action at different skill levels. Res. Q. Exerc. Sport 74, 257-269. doi: 10.1080/02701367.2003.106 09090

Castagna, C., Impellizzeri, F. M., Rampinini, E., D’Ottavio, S., and Manzi, V. (2008). The Yo-Yo intermittent recovery test in basketball players. J. Sci. Med. Sport 11, 202-208. doi: 10.1016/j.jsams.2007.02.013

Csapo, P., Avugos, S., Raab, M., and Bar-Eli, M. (2015). How should "hot" players in basketball be defended? The use of fast-and-frugal heuristics by basketball coaches and players in response to streakiness. J. Sports Sci. 33, 1580-1588. doi: $10.1080 / 02640414.2014 .999251$

Csapo, P., and Raab, M. (2014). Hand down, man down." Analysis of defensive adjustments in response to the hot hand in basketball using novel defense metrics. PLoS One 9:e114184. doi: 10.1371/journal.pone.0114184

Eloranta, V. (1996). Effect of postural and load variation on the coordination of the leg muscles in concentric jumping movement. Electromyogr. Clin. Neurophysiol. $36,59-64$.

Erculj, F., and Supej, M. (2009). Impact of fatigue on the position of the release arm and shoulder girdle over a longer shooting distance for an elite basketball player. J. Strength Cond. Res. 23, 1029-1036. doi: 10.1519/JSC.0b013e3181a $07 \mathrm{a} 27$

Fleiss, J. L. (1991). Statistical Methods for Rates and Proportions. New York, NY: JohnWiley \& Sons.

Forestier, N., and Nougier, V. (1998). The effects of muscular fatigue on the coordination of a multijoint movement in human. Neurosci. Lett. 252, 187-190. doi: 10.1016/S0304-3940(98)00584-9

Francavilla, C. V., Sessa, F., Salerno, M., Albano, G. D., Villano, I., Messina, G., et al. (2018). Influence of football on physiological cardiac indexes in professional and young athletes. Front. Physiol. 9:153. doi: 10.3389/fphys.2018.00153

Garrett, W. E., and Kirkendall, D. T. (2000). Exercise and Sport Science. Philadelphia, PA: Lippincott Williams \& Wilkins.

Gheller, R. G., Dal Pupo, J., Ache-Dias, J., Detanico, D., Padulo, J., and dos Santos, S. G. (2015). Effect of different knee starting angles on intersegmental

\section{ACKNOWLEDGMENTS}

The authors wish to thank all the players who volunteered in this study, their athletic trainer Giacomo Dascanio, and Ms. Dinah Olswang for english editing.

coordination and performance in vertical jumps. Hum. Mov. Sci. 42, 71-80. doi: 10.1016/j.humov.2015.04.010

Ibáñez, S. J., Feu, S., Cañadas, M., Parejo, I., and García, J. (2009). Shot differences between professional (ACB) and amateur (EBA) basketball teams. Multifactorial study. Rev. Psicol. Deporte 18, 313-317.

Karvonen, M. J., Kentala, E., and Mustala, O. (1957). The effects of training on heart rate; a longitudinal study. Ann. Med. Exp. Biol. Fenn. 35, 307-315.

Lephart, S. M., Riemann, B. L., and Fu, F. H. (2000). Proprioception and Neuromuscular Control in Joint Stability. Champaign, IL: Human Kinetics.

Llorca-Miralles, J., Piñar López, M. I., Cárdenas Vélez, D., Sánchez-Delgado, G., and Perales, J. C. (2013). Basketball training influences shot selection assessment: a multi-attribute decision-making approach. Rev. Psicol. Deporte $22,223-226$.

Lyons, M., Al-Nakeeb, Y., and Nevill, A. (2006). The impact of moderate and high intensity total body fatigue on passing accuracy in expert and novice basketball players. J. Sports Sci. Med. 5, 215-227.

McInnes, S. E., Carlson, J. S., Jones, C. J., and McKenna, M. J. (1995). The physiological load imposed on basketball players during competition. J. Sports Sci. 13, 387-397. doi: 10.1080/02640419508732254

Miller, S., and Bartlett, R. (1996). The relationship between basketball shooting kinematics, distance and playing position. J. Sports Sci. 14, 243-253. doi: 10. 1080/02640419608727708

Mokou, E., Nikolaidis, P. T., Padulo, J., and Apostolidis, N. (2016). The acute effect of exercise intensity on free throws in young basketball players. Sport Sci. Health 12, 227-232. doi: 10.1007/s11332-016-0279-8

Newell, K. M., and Vaillancourt, D. E. (2001). Dimensional change in motor learning. Hum. Mov. Sci. 20, 695-715. doi: doi:10.1016/S0167-9457(01)00073-2

Nikolaidis, P., Calleja-González, J., and Padulo, J. (2014). The effect of age on positional differences in anthropometry, body composition, physique and anaerobic power of elite basketball players. Sport Sci. Health 10, 225-233. doi: 10.1007/s11332-014-0198-5

Nikolaidis, P. T., Asadi, A., Santos, E. J., Calleja-González, J., Padulo, J., Chtourou, H., et al. (2015). Relationship of body mass status with running and jumping performances in young basketball players. Muscles Ligaments Tendons J. 5, 187-194. doi: 10.11138/mltj/2015.5.3.187

Okazaki, V. H. A., Rodacki, A. L. F., Dezan, V. H., and Sarraf, T. A. (2007). Coordenação do arremesso de jump no basquetebol de crianças e adultos. Rev. Bras. Biomech. 1, 15-22.

Okazaki, V. H. A., Rodacki, A. L. F., and Satern, M. N. (2015). A review on the basketball jump shot. Sports Biomech. 14, 190-205. doi: 10.1080/14763141.2015. 1052541

Ortega, E., Cárdenas, D., Sainz De Baranda, P., and Palao, J. M. (2006). Analysis of the final actions used in basketball during formative years according to player's position. J. Hum. Mov. Stud. 50, 421-437.

Padulo, J., Attene, G., Migliaccio, G. M., Cuzzolin, F., Vando, S., and Ardigò, L. P. (2015a). Metabolic optimisation of the basketball free throw. J. Sports Sci. 33, 1454-1458. doi: 10.1080/02640414.2014.990494

Padulo, J., Laffaye, G., Haddad, M., Chaouachi, A., Attene, G., Migliaccio, G. M., et al. (2015b). Repeated sprint ability in young basketball players: one vs. two changes of direction (Part 1). J. Sports Sci. 33, 1480-1492. doi: 10.1080/ 02640414.2014 .992936

Padulo, J., Bragazzi, N. L., Nikolaidis, P. T., Dello Iacono, A., Attene, G., Pizzolato, F., et al. (2016). Repeated sprint ability in young basketball players: multi-direction vs. one-change of direction (Part 1). Front. Physiol. 7:133. doi: 10.3389/fphys.2016.00133

Petito, A., Altamura, M., Iuso, S., Padalino, F. A., Sessa, F., D’Andrea, G., et al. (2016). The relationship between personality traits, the 5HTT polymorphisms, and the occurrence of anxiety and depressive symptoms in elite athletes. PLoS One 11:e0156601. doi: 10.1371/journal.pone.0156601 
Rodacki, A. L. F., Fowler, N. E., and Bennett, S. J. (2002). Vertical jump coordination: fatigue effects. Med. Sci. Sports Exerc. 34, 105-116. doi: 10.1097/ 00005768-200201000-00017

Sessa, F., Chetta, M., Petito, A., Franzetti, M., Bafunno, V., Pisanelli, D., et al. (2011). Gene polymorphisms and sport attitude in Italian athletes. Genet. Test. Mol. Biomarkers 15, 285-290. doi: 10.1089/gtmb.201 0.0179

Skinner, B. (2012). The problem of shot selection in basketball. PLoS One 7:e30776. doi: 10.1371/journal.pone.0030776

Temprado, J., Della-Grasta, M., Farrell, M., and Laurent, M. (1997). A noviceexpert comparison of (intra-limb) coordination subserving the volleyball serve. Hum. Mov. Sci. 16, 653-676. doi: 10.1016/S0167-9457(97)0 0014-6

Zagatto, A. M., Ardigò, L. P., Barbieri, F. A., Milioni, F., Dello Iacono, A., Camargo, B. H. F., et al. (2017). Performance and metabolic demand of a new repeated-sprint ability test in basketball players: does the number of changes of direction matter? J. Strength Cond. Res. 31, 2438-2446. doi: 10.1519/JSC. 0000000000001710

Conflict of Interest Statement: The authors declare that the research was conducted in the absence of any commercial or financial relationships that could be construed as a potential conflict of interest.

Copyright $\odot 2018$ Padulo, Nikolaidis, Cular, Dello Iacono, Vando, Galasso, Lo Storto and Ardigo. This is an open-access article distributed under the terms of the Creative Commons Attribution License (CC BY). The use, distribution or reproduction in other forums is permitted, provided the original author(s) and the copyright owner(s) are credited and that the original publication in this journal is cited, in accordance with accepted academic practice. No use, distribution or reproduction is permitted which does not comply with these terms. 